\title{
Recruitment Challenges for Studies of Deep Brain Stimulation for Treatment-Resistant Depression
}

This article was published in the following Dove Press journal:

Neuropsychiatric Disease and Treatment

\author{
Rajamannar Ramasubbu ${ }^{1-4}$ \\ Sandra Golding ${ }^{1,2}$ \\ Kimberly Williams' \\ Aaron Mackie' \\ Glenda MacQueen (D) 1,3,4 \\ Zelma HT Kiss ${ }^{\text {I-4 }}$
}

'Department of Psychiatry, University of Calgary, Calgary, AB, Canada;

${ }^{2}$ Department of Clinical Neuroscience,

University of Calgary, Calgary, AB,

Canada; ${ }^{3}$ Mathison Centre for Mental

Health Research \& Education, University

of Calgary, Calgary, AB, Canada;

${ }^{4}$ Hotchkiss Brain Institute, University of

Calgary, Calgary, AB, Canada
Correspondence: Rajamannar Ramasubbu Department of Psychiatry/Clinical Neurosciences, University of Calgary,

Mathison Centre for Mental Health Research and Education, TRW Building, Room 4D64, 3280 Hospital Drive NW,

Calgary, Alberta, T2N4Z6

Tel + 4032106890

Fax +1 4032109114

Email rramasub@ucalgary.ca
Introduction: Deep brain stimulation (DBS) is currently an investigational treatment for treatment-resistant depression (TRD). There is a need for more DBS trials to strengthen existing evidence of its efficacy for both regulatory and clinical reasons. Recruitment for DBS trials remains challenging due to unproven efficacy in sham-controlled DBS trials, invasive nature of the intervention and stringent eligibility criteria in patient selection. Here, we examined the referral patterns and reasons for exclusion of subjects in our DBS trial.

Methods: Data were collected from all patients who expressed interest in participating in a DBS study involving subcallosal cingulate region from 2014 to 2016 . Referral sources were categorized as either self-referral or professional referral. Evaluation for eligibility was performed in three stages; initial contact, brief telephone assessment, and in-person psychiatric evaluation. The reasons for exclusion were documented. Descriptive and inferential statistics were used for analysis.

Results: Of the 225 patients who contacted us initially, 22 (9.2\%) underwent DBS surgery. Self-referral was higher than the referral from professionals ( $72 \%$ versus $28 \%, \mathrm{P}<0.0001)$. However, the acceptance rate for surgery was higher among the professional referrals than from self-referrals ( $40 \%$ versus $15 \%, \mathrm{P}=0.03$ ). The common reasons for exclusion were selfwithdrawal $(38.4 \%)$, residing out of province or country $(26.1 \%)$ and psychiatric/medical comorbidity $(21.7 \%)$.

Conclusion: These findings provide insight into DBS candidacy for future TRD trials. It suggests a need for comprehensive recruitment strategies including active engagement of patients and professionals throughout trials, and effective referral communication with education to optimize recruitment for future DBS trials.

Keywords: patient recruitment, deep brain stimulation, treatment-resistant depression, clinical trials, subcallosal cingulate region

\section{Introduction}

Deep brain stimulation (DBS) is an investigational therapy for patients with advanced treatment-resistant depression (TRD). Numerous open-label trials involving several brain targets (subcallosal cingulate region, ventral capsule/ventral striatum, anterior limb of the internal capsule, superolateral branch of the median forebrain bundle) document the safety and efficacy of DBS treatment for TRD. ${ }^{1}$ Despite these initial successes, two large industry-sponsored multicenter shamcontrolled trials produced negative results in futility analyses ${ }^{2,3}$ and also failed to meet their endpoints. These two trials are major setbacks for the advancement of DBS research in TRD and also for regulatory and clinical care. The DBS research community has spent countless hours debating the cause of these apparent trial 
failures suggesting reasons such as poor trial design, suboptimal electrode targeting, use of restrictive stimulation algorithms, inappropriate selection of patients due to lack of predictive biomarkers, shorter trial duration, and less specific outcome measures. ${ }^{4}$ The lack of understanding of DBS mechanisms in the treatment of TRD and premature randomized trial design before there are reasonable hypotheses of mechanisms are most likely to blame for poor results.

To overcome the methodological shortcomings learnt from these previous trials, several groups have suggested adaptive trial design, including diffusion tensor imaging (DTI) based precise targeting ${ }^{5,6}$ and biomarker informed patient selection. ${ }^{7,8}$ Others have proposed enriched sample trial designs using withdrawal or discontinuation of DBS after optimization of stimulation and clinical outcomes. ${ }^{9,10}$ To move forward in this field, new DBS trials are needed if they are to contribute to new scientific knowledge for the development of DBS therapy for TRD. While DBS for obsessive compulsory disorder (OCD) was given humanitarian device exemption (HDE) by the Food and Drug Administration (FDA) in 2009, DBS for TRD is not approved. ${ }^{4}$ Regulatory agencies such as the FDA require valid scientific evidence from well-controlled investigations to partially controlled trials in order to approve the use of DBS system for TRD. Furthermore, DBS is an expensive treatment and its estimated cost per qualityadjusted year is about 165,000 USD which is much higher than the estimated cost for Parkinson disease. ${ }^{11}$ In fact, DBS for TRD would need to achieve higher response rates of $\approx 70 \%$, rather than the historical response rates of 40 to $-50 \%$ to justify its cost. ${ }^{11}$ Therefore, future DBS studies are essential to guiding improvement and maximizing the clinical outcomes needed to justify DBS cost-effectiveness for wider clinical use.

Effective recruitment is pivotal for the success of DBS studies. Recruitment for DBS trials in patients with TRD remains challenging due to stringent eligibility criteria, invasive nature of the treatment and unproven efficacy in sham-controlled trials. ${ }^{12}$ Insights from previous DBS trials on recruitment may be instructive for future trials. Only one report has examined recruitment of patients for a subcallosal cortex (SCC) DBS trial in TRD. ${ }^{13}$ This study found that among 1098 patients screened only 2\% were eligible for DBS surgery and a lack of adequate electroconvulsive treatment (ECT) was the most common cause of exclusion. They reported a large proportion of inappropriate referrals which could increase the cost of recruitment and delay completion of studies in the future. Furthermore, the low percentage of eligible patients and large volume of inappropriate referrals reflect the complexities of the recruitment process. Other single-center and multicenter studies discuss the low recruitment rate as a barrier for the success of DBS trials in TRD. ${ }^{12,14}$ Therefore, there is a growing need to better understand the sources of difficulty in recruitment. We reviewed the referral patterns for our randomized controlled DBS trial for TRD at a tertiary care center in Canada and examined the sources of referral and reasons for exclusion.

\section{Methods}

This study included data from all subjects who expressed interest in participating in our DBS for TRD investigational trial funded through an Alberta Innovates Health Solutions (AIHS) Collaborative Research \& Innovation Opportunities (CRIO) grant. ${ }^{15}$ The study was conducted in a single academic tertiary care center, at the Foothills Hospital affiliated with the University of Calgary in Calgary, Alberta, Canada. The study protocol was approved by the University of Calgary's Conjoint Ethics Review Board. The details of the trial design, methodology and results of this study were described elsewhere. ${ }^{15}$ All participants provided informed consent and this study was conducted in accordance with the Declaration of Helsinki.

\section{Recruitment Planning and Strategies}

Our strategies to achieve recruitment targets evolved in three phases. First, we performed a DBS trial feasibility pilot study at our site from 2008 to $2012^{16}$ before this major trial was initiated. In the feasibility trial, recruitment was a major challenge as it took nearly three years to recruit four patients. The major reason for poor recruitment was that the recruitment source was limited only to psychiatrists working in three teaching hospitals in Calgary.

For our main trial, we expanded recruitment strategies to involve community psychiatrists, family physicians as well as patients/families throughout the province. During our protocol development, we engaged patient advocacy groups such as the Organization for Bipolar Affective Disorder (OBAD), the Mood Disorder Association, and the Canadian Mental Health Association. We also engaged with local psychiatrists in leadership positions. These groups' contributions were incorporated into the development of the grant proposal and trial protocol. For instance, 
patient advocacy groups advocated to include individual cognitive behavioral therapy (CBT) instead of group CBT for the CBT add-on treatment component of our trial. Third, after receiving the grant, we held several meetings with patient advocacy groups, patients with lived experience, and clinical psychiatrists in and around the city. We communicated important messages related to the study and the clinical value to them. In addition, we addressed the questions and concerns related to the surgery, devices, continuous stimulation and possible side effects. We established a website and distributed posters at every hospital and some community centers in Calgary. Recruitment pamphlets were sent to many psychiatrists' offices by e-mail and post. There was also a press release and media interviews and a surge in self-referred patients who were always looking for new treatments followed these events. To improve the literacy of DBS in TRD and referral process of the study, several educational presentations were made in major hospitals and at local conferences. Informal meetings were held with inpatient psychiatrists and staff at Calgary tertiary care mood clinic.

\section{Recruitment Procedure}

Evaluation of subjects for eligibility was performed in three stages. Initial contact (stage I) was a preliminary screen performed by the registered nurse $(\mathrm{RN})$ research coordinator who assessed each participant via telephone or e-mail to discuss the study. If no obvious exclusion criteria were identified and the participant wished to continue then they proceeded to a comprehensive telephone screen (stage II), again performed by the same RN coordinator with a semi-structured questionnaire that involved specific questions (Supplementary Data). This stage II confirmed initial inclusion criteria including a self-reported diagnosis of major depressive disorder (MDD) or bipolar type depression. The participant also needed to have current Alberta health insurance coverage or be willing to relocate and qualify for provincial insurance. This phone screen also included detailed information regarding a patients' psychiatric and medical history. All phone screens were conducted by the same research coordinator to ensure reliability and consistency between screens.

A study psychiatrist reviewed completed phone screens. If still deemed eligible, participants were invited to come for the stage III in-person psychiatric screening visit. During this assessment, the psychiatrist assessed participants to ensure they met study requirements including a diagnosis of TRD and current major depressive episode (MDE). Complete exclusion and inclusion criteria were reviewed during this stage. After the in-person psychiatric screen if the participant was still deemed eligible, then a second, independent psychiatric evaluation was conducted at a different time to confirm eligibility.

If assessed to be eligible based on two independent psychiatric evaluations, subjects went on to neurosurgical evaluation, magnetic resonance imaging, and laboratory investigations. Participants' medical records including previous psychiatric records were reviewed. Participants continued to be excluded if they were found to not be medically fit for neurosurgery or chose to withdraw on their own.

\section{Inclusion and Exclusion Criteria}

Details of eligibility criteria are provided in Table 1 and summarized in our previous outcome paper. ${ }^{15}$ Treatment resistance was defined as having trialed at least four antidepressant classes of medications and psychotherapy. If they had a diagnosis of bipolar depression, the participant also had to have been on concomitant mood stabilizers. Beyond medication treatment and psychotherapy, participants needed to have had failure, intolerance, and contraindication or be unwilling to accept ECT.

Exclusion criteria included psychiatric comorbidities, such as depressed patients with a psychiatric diagnosis other than MDD, bipolar I disorder (BPI) or bipolar II disorder (BPII) (Table 1). Other exclusion criteria were participants who did not have Alberta health care insurance or were unwilling to relocate to Alberta for the duration of the study. Participants who did not attend follow up to any stage of screening did not respond to e-mails/phone calls or who stated they were not interested in the study were excluded and labeled as "patient withdrawal".

Statistical analyses were performed using Stata (StataCorp, version 13.1) and Excel (Microsoft version 15.30). Descriptive statistics were calculated for each reason for exclusion for the entire group. The differences in referral rates between sources were described. Nonparametric tests (chi-square) and parametric tests (student t-tests) were used to assess differences between categorical and continuous variables, respectively. A p-value less than or equal to 0.05 was chosen a priori as the threshold for statistical significant. 
Table I Inclusion and Exclusion Criteria

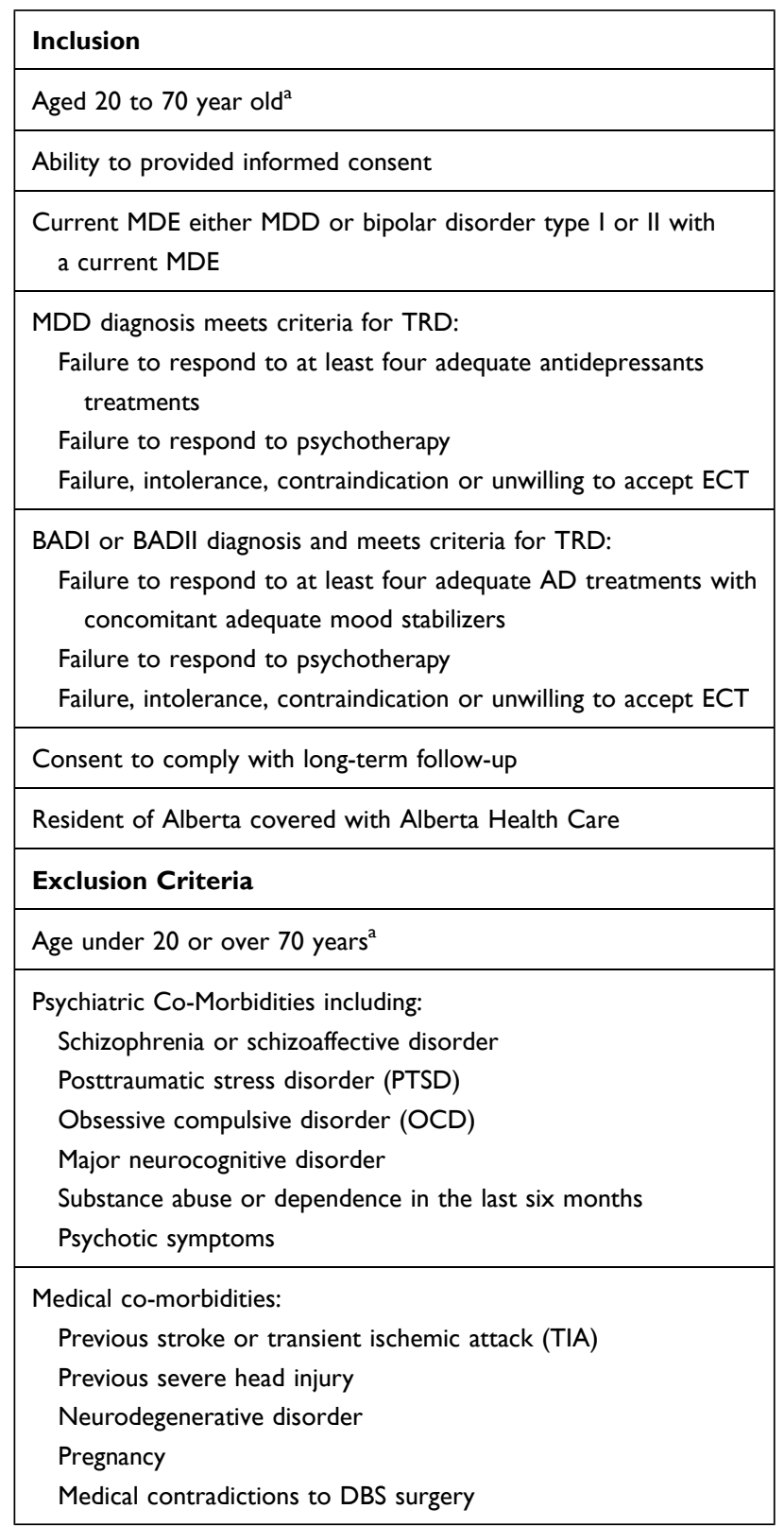

Note: a During the study the age of inclusion was increased to 70 years from 60 years.

Abbreviations: $A D$, antidepressant; MDE, major depressive episode; MDD, major depressive disorder; TRD, treatment-resistant depression; ECT, electroconvulsive therapy; BADI, bipolar I disorder; BADII, bipolar II disorder.

\section{Results}

Initially, 225 subjects made contact because they were interested in the DBS study. Figure 1 displays the flow diagram of the study including the number of participants at each stage of screening. Interested subjects came from Alberta, other provinces within Canada, Europe and the United States of
America. Out of 225 subjects who contacted the study team, 121 subjects $(54.2 \%)$ were excluded or withdrawn in stage I. Of the 104 participants who underwent the comprehensive phone screen (stage II), 50 patients (48.1\%) were excluded. Of the 54 participants who underwent psychiatric assessment (stage III) 32 (59.3\%) were excluded leaving 22 participants.

Table 2 lists the reasons that participants were excluded. The most common reason for exclusion in stage I was participant self-withdrawal. Overall within all stages, $77(37.9 \%)$ of the 203 patients excluded were due to lack of interest in participating/patient withdrawal. The other reasons included living outside the province/country $(\mathrm{N}=53 ; 26.1 \%)$, and having a psychiatric and/or medical comorbidity $(\mathrm{N}=45 ; 22.2 \%)$. Only 14 patients $(6.9 \%)$ were excluded due to inadequate treatment.

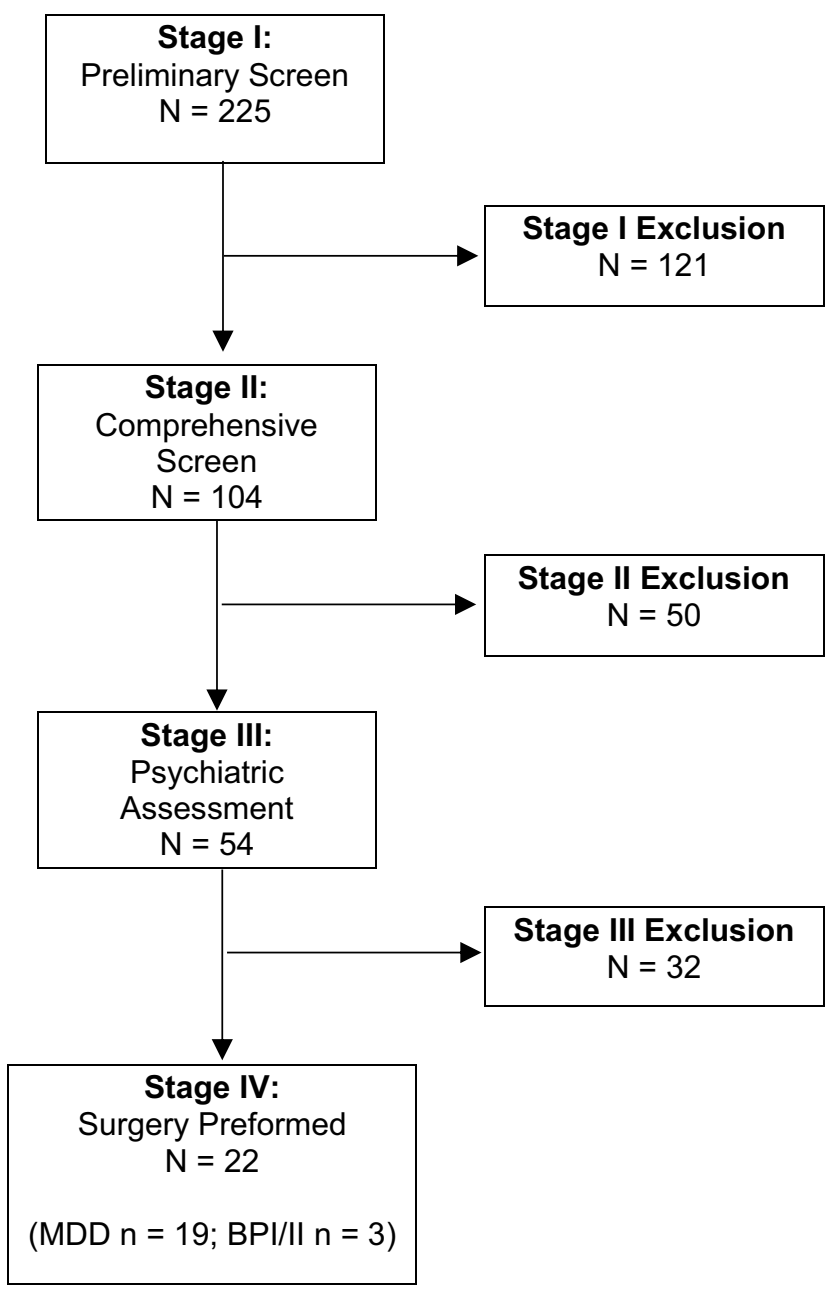

Figure I Flow chart of patient screening for eligibility by stage. 
Table 2 Reasons for Exclusion by All Stages ${ }^{\dagger}$

\begin{tabular}{|l|l|l|}
\hline Reason for Exclusion & N & $\%$ \\
\hline Inadequate treatment & 14 & 6.9 \\
\hline Over age limit & $\mathrm{II}$ & 5.4 \\
\hline Out of country & 30 & 14.8 \\
\hline Out of province & 23 & 11.3 \\
\hline Self-withdrawal & 77 & 37.9 \\
\hline Medical co-morbidity & 12 & 5.9 \\
\hline Psychiatric co-morbidity & 33 & 16.3 \\
\hline Misdiagnosis & 2 & 1.0 \\
\hline Suicide & $\mathrm{I}$ & 0.5 \\
\hline TOTAL & 203 & 100.0 \\
\hline
\end{tabular}

Notes: ${ }^{\dagger}$ Numbers reported are mutually exclusive. Subjects who had more than one exclusion cause were included under one reason in the following order: out of province/out of country, self-withdrawal, age, then inadequate treatment, the medical co-morbidity and finally psychiatric co-morbidity.

Referral source, age, and sex information was not collected for all participants in stage I. For participants who proceeded to the stage II phase, the majority were self-referrals $(\mathrm{N}=75$, $72 \%)$, rather than health professional referrals $(\mathrm{N}=29,28 \%)$ (Figure 2) and this represented a significant difference using univariate Chi-square test $\left(\chi^{2}=20.35, \mathrm{p}<0.0001\right)$ (Table 3). However, participants were more likely to be accepted for surgery if they were referred by a professional $\left(\chi^{2}=5.46, \mathrm{p}=\right.$ 0.02). There was no significant difference in referral source by age $(p=0.79)$ or gender $(p=0.11)$ (Table 3$)$. Self-referred patients who underwent DBS surgery showed comparable clinical efficacy and adverse effects compared to professionally referred patients with DBS surgery (Table 4). Furthermore, trial compliance of self-referred patients was equivalent to professionally referred patients.

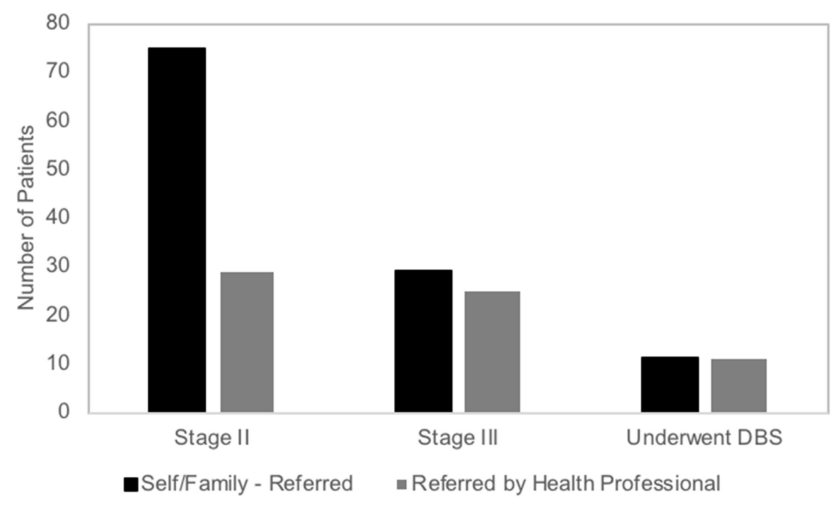

Figure 2 Source of referral at each stage of screening for eligibility and proportion undergoing DBS surgery.
Table 3 Comparison of Self- versus Professional Referral Participants for Stage II and III

\begin{tabular}{|l|l|l|}
\hline Demographics & $\begin{array}{l}\text { Self- } \\
\text { Referral }\end{array}$ & $\begin{array}{l}\text { Professional } \\
\text { Referral }\end{array}$ \\
\hline Number of Patients (n, \%) & $75(72 \%)$ & $29(28 \%)^{*}{ }^{\dagger}$ \\
\hline Age (mean \pm SD in years) & $46.29 \pm 13.02$ & $45.92 \pm 12.57$ \\
\hline Gender (\% male, \% female) & $40 \%, 60 \%$ & $55 \%, 45 \%$ \\
\hline Excluded (n, \%) & $64(85 \%)$ & $18(62 \%)$ \\
\hline $\begin{array}{l}\text { Number accepted for surgery } \\
\text { (n, \%) }\end{array}$ & $11(15 \%)$ & $11(38 \%)^{*},{ }^{\dagger \dagger}$ \\
\hline
\end{tabular}

Notes: ${ }^{*} \mathrm{p}<0.05$; ${ }^{\dagger}$ univariate Chi-square test; ${ }^{\dagger+}$ bivariate Chi-square test.

\section{Discussion}

New DBS trials for TRD are essential to determine the efficacy, safety, mechanisms of stimulation and best pathways to optimize treatment outcomes. However, the investigators involved in DBS trials for TRD face significant

Table 4 DBS Outcome by Source of Referral

\begin{tabular}{|c|c|c|}
\hline Outcomes & $\begin{array}{l}\text { Professional } \\
\text { Referral } \\
\mathrm{N}=\text { I I }\end{array}$ & $\begin{array}{l}\text { Self-Referral } \\
N=I \mid\end{array}$ \\
\hline \multicolumn{3}{|l|}{ Trial Compliance } \\
\hline $\begin{array}{l}\text { *Missed appointments (number } \\
\text { of patients) }\end{array}$ & $6(n=4)$ & $5(n=3)$ \\
\hline \multicolumn{3}{|l|}{ Clinical outcome } \\
\hline \multicolumn{3}{|c|}{ Responders ( $>50 \%$ reduction in HDRS scored from the baseline) } \\
\hline 6 months & $6(55 \%)$ & $4(36 \%)$ \\
\hline 12 months & $5(45 \%)$ & $6(55 \%)$ \\
\hline \multicolumn{3}{|l|}{ Serious Adverse events } \\
\hline Suicide & I (during the trial) & I (after the trial) \\
\hline $\begin{array}{l}\text { Hospitalization (due to increase } \\
\text { suicidality depression) }\end{array}$ & $2(20 \%)$ & 0 \\
\hline \multicolumn{3}{|l|}{ **Adverse events } \\
\hline Worsening depression & I (I0\%) & $3(25 \%)$ \\
\hline Worsening anxiety & $3(30 \%)$ & $4(33 \%)$ \\
\hline Subjective memory impairment & $2(20 \%)$ & $2(17 \%)$ \\
\hline Increased anger & $3(30 \%)$ & 0 \\
\hline Increased insomnia & $\mathrm{I}(10 \%)$ & I (8\%) \\
\hline
\end{tabular}

Notes: *Patient died by suicide at 3 month was excluded. **Adverse events were temporary and improved with adjustment of stimulation parameter and stimulation mode. 
recruitment barriers. The available literature provides little information on factors that impact the actual recruitment and enrolment of patients with TRD for DBS trials. Here we report source of referral (professional versus selfreferral) and causes of exclusion. To advance this therapy, it is critical to use the most time and cost-efficient recruitment strategies for future DBS trials.

Consistent with a previous study, ${ }^{13}$ we found that selfreferral rates were higher than professional referrals. Yet enrollment rates for surgery were higher from professionals than from self-referral because exclusion rates were lower. The observed higher rate of self-referral for DBS is promising despite the fact that patients with chronic TRD may harbor negative beliefs about the effectiveness of treatment because of their previous experience of multiple treatment failures. Negative expectations of treatment outcome are considered one of the personal barriers to seeking treatment. ${ }^{17}$ Higher rates of selfreferral for an invasive treatment are intriguing but could be partly explained by the findings that depressed patients experiencing severe symptoms and distress, with longer duration of symptoms and lower perceived personal stigma were more likely to seek treatment ${ }^{17-19}$. Although these patients failed to improve with multiple trials of antidepressant treatments, they were highly motivated to try new invasive treatments like DBS suggesting that the previous experience of receiving treatment for depression had minimal impact on help-seeking behavior ${ }^{20}$ and treatment failures did not deter treatment-seeking in this cohort. Interestingly, compared to professional referrals more self- referred patients with TRD sought DBS treatment in a previous study ${ }^{13}$ as well as in our study although these patients were less motivated, and hopeless in their life due to TRD. It is possible that the prolonged depressed mood may be a key reason for DBS self-referral in TRD patients which needs further evaluation.

Self-referral is a successful recruitment method for randomized controlled trials in depression. ${ }^{21}$ This method could be complimentary or adjunct to a professional referral route. A self-referral approach could reach more TRD patients in the community who may have not received adequate treatment or have given up on medical options provided by a psychiatrist or family physician. It circumvents the professional referral and can increase access for ethnic minorities who are more difficult to engage in research. ${ }^{21}$ In our center, recruitment planning involved strategies to increase self-referral as well as clinician referral for any depression treatment trials regardless of the nature of treatment. For non-invasive trials, we have previously successfully recruited from self-referred university students and staff with nonresistant or drug naïve MDD through posters and local advertisements in University/Hospital websites. Since DBS is an invasive and experimental treatment targeting advanced TRD, we anticipated more challenges in recruitment. Hence, we employed community and hospital-based approaches and extended our efforts to province-wide recruitment. We also engaged patient advocacy groups and professionals periodically for this DBS trial.

There are challenges in the recruitment for invasive treatment from self-referrals. As shown in our study, large proportion of patients recruited by self-referral did not meet the criteria for inclusion in the study. Additionally, the amount of time needed to screen and perform assessments for those self-referred took longer than anticipated. Successful recruitment from self-referral methods may require additional budgeting and resources. From an ethical perspective, self-referred patients for this DBS trial needed greater attention in obtaining informed consent than in non-invasive trials involving self-referred patients. The study psychiatrist had to ensure that desperation should not affect decision-making capacity or voluntariness of patients participating in the DBS study. ${ }^{22}$ Therefore, the study psychiatrist (RR) and nurse coordinator $(\mathrm{SG})$ took significant time to explain the risks and benefits to patients and their family members (if available) regarding both the surgery and the randomization protocol. The safety and efficacy of pulse-width stimulus optimization were based on the principles of clinical equipoise. ${ }^{23}$ Since this was an active comparative trial without sham control arm, there were no ethical concerns about using placebo or inactive treatment in this trial. The patients were assured of receiving active stimulation regardless to which arm they were randomized. We also clarified all their questions, concerns and corrected the misperceptions or misplaced expectations regarding DBS.

Our results suggest that self-referred patients were more likely to withdraw from the study. Although not all patients gave reasons for voluntary withdrawal, those who reported reasons indicated that some did not know they could receive non-invasive treatment options for TRD, such as repetitive Transcranial Magnetic Stimulation (rTMS) and ketamine infusion at the time of their recruitment. Others realized their inability to commit for monthly follow-ups. A few participants withdrew because of major 
negative life events such as marital breakdown or refusal of family members (spouse or children) to support DBS treatment.

Despite the drawbacks, our study suggests that the selfreferral route was crucial to complete this study in the specified time. $50 \%$ of patients (11 out of 22) operated for DBS surgery were self-referred. Additionally, the trial compliance and clinical outcomes were comparable between self-referred and professionally referred patients. Suicide might have been prevented in two patients from the professionally referred group by timely intervention of their psychiatrists and hospitalization. Lack of adequate psychiatric care outside the trial might have contributed to one self-referred patient who died by suicide as this patient had great difficulty in finding a psychiatrist after moving to another province after the completion of the trial. This emphasizes the need for ongoing psychiatric care indefinitely for these patients. Other adverse events such as increasing anxiety, memory disturbances were equally distributed between the groups. There was no difference in the number of patients who experienced worsening of depression when hospitalization due to depression was included in the metrics. The incidence of increased anger was reported only in the professionally referred group which was stimulation-related and controlled with adjustment of stimulation parameters.

Referrals from psychiatrists appear to be the most effective means of recruiting good candidates into DBS trials for TRD. The high percentage of appropriate referrals for surgery from their referrals may reflect their better understanding of TRD and the protocol of the study. However, the low level of referral rate from physician for DBS trials for TRD is also a matter of concern. ${ }^{13}$ Although exact reasons remain unknown, we can offer several possible explanations for the lack of physicians' engagement in this DBS trial. Previous research on physician referral barriers in clinical trials has shown that the lack of confidence in the efficacy and safety of the treatment, the complexity of the protocol including stringent exclusion criterion, feeling unsure about eligibility, referral process burden (lack of time and structural/administrative support for referrals), mistrust of institutions and researchers, fear of losing patients, and lack of clinical trial awareness may influence physician engagement in the referral process. ${ }^{24,25}$ However, evidence-based information on potential barriers to psychiatrists' referral of TRD patients for DBS studies remains limited. One recent study evaluated the attitudes and perceptions of 35 psychiatrists and
64 psychiatric resident physicians pertaining to DBS and neuroablation procedures for treating psychiatric disorders. ${ }^{26}$ In this study, only $11.4 \%$ had ever made a referral for DBS/ablative surgery. The reported potential barriers for referral to psychiatric neurosurgery included lack of knowledge of the neuronal basis of the illness, technical procedures, evidence-informed outcomes, side effects, referral process, and patient and family resistance. Additionally, some psychiatrists felt that both DBS/ablative surgery were dangerous and unethical with potential for abuse by professionals. While we did not examine the potential barriers for psychiatrist referral in our study, these previously identified barriers for professional referrals should be considered and mitigated to improve recruitment. To improve professional referrals better information is needed for both practicing physicians and medical trainees to make informed decisions regarding referral based on risks and benefits of DBS. ${ }^{26} \mathrm{~A}$ comprehensive recruitment plan as proposed by the Clinical Trial Transformation Initiative (CTTI) encompassing the continuous engagement of stakeholders and implementing effective communication of trial details will likely improve recruitment in future studies. ${ }^{27}$ In this study, direct engagement with physician groups stopped at the end of the first year. In the following years of the study (years two through four), we communicated to psychiatrists only through e-mail and mail which may have been, in hindsight, a less effective communication strategy than direct meetings.

Movement disorder DBS referral is a very different process. Professional referrals from movement disorder specialists, general neurologists, and primary care physicians were reported to be much higher than self-referral $(87 \%$ vs $11 \%){ }^{28}$ Lessons can be learned from the insights gained from DBS trials for movement disorders to improve professional referrals for DBS trials of TRD. Since DBS is an approved treatment for movement disorders, these patients are directly referred for DBS from their physicians whereas DBS for TRD is an experimental treatment and professionals are reluctant to refer their patients for experimental treatment. Second, consistent with other academic centers, our centre has a specialized movement disorder clinic where all complex patients attend this specialty clinic by the time they are advanced enough to require surgery. Therefore, only movement disorder neurologists refer for DBS surgery. These specialized clinics are the most important referral source for good candidates for movement disorder surgery. Along 
similar lines, establishing a specialized clinic for TRD or registry for patients with TRD may improve recruitment for DBS surgery from academic centers. In fact, specialized clinics for TRD are established in several academic centers, which provide consultation as well as opportunities for patients to participate in novel experimental treatment studies. Some suggested specialized neuromodulation units are providing both non-invasive and invasive neuromodulation treatments for psychiatric conditions such as TRD and obsessive-compulsive disorder (OCD) and offering a comprehensive pre-treatment selection and post-treatment care. ${ }^{29}$ In our centre, there is no specialized clinic for patients with TRD or comprehensive neuromodulation treatment unit. Patients with unipolar TRD usually attend several community mental health clinics, private psychiatrists, family doctors and hospitals for chronic mental illnesses. Academic centers which offer invasive and non-invasive neuromodulatory treatments for TRD through clinical or research programs should have the specialized clinics to improve the professional referral for DBS for TRD.

In single-center studies, site-specific recruitment barriers including the availability and accessibility of other competing therapies for TRD such as vagal nerve stimulation (VNS), nasal des-ketamine or ketamine infusion, TMS (transcranial magnetic stimulation), and related competing interests of the investigators providing these therapies may affect the recruitment and referral patterns. VNS therapy is approved as adjunct therapy of TRD in Canada, Europe, USA. ${ }^{30}$ However, in our center, it is used only for intractable epilepsy not for TRD. TMS and ketamine therapies for clinical purposes were not readily available at our center during this trial. A concurrent research study involving TMS for depression included only depressed patients with mild resistance (failure to 1 or 2 antidepressant medications) which did not affect the recruitment of patients with severe resistance (failure to 4 treatment trials) for this DBS trial.

Multicenter studies are suggested if recruitment targets are too high to achieve within a specified time period in a single center. Despite this, a multicenter European study was prematurely stopped because of poor recruitment as the study teams could recruit only nine of 60 subjects in two years. ${ }^{12}$ While our study was single center, we achieved $88 \%(n=23)$ of our targeted sample of 25 subjects in three years, perhaps because our target sample was small. ${ }^{12,31}$ Although our recruitment success could be partly related to our recruitment planning and strategies, and active engagement of end users in the early stages, the professional referrals were still very low. Besides general reasons for poor referral rates mentioned previously, we may also have failed to selectively engage the psychiatrists who are critical about surgical treatment of depression during the study communications. The psychiatrists who attended the study communication meetings were predominantly those who were open to surgical interventions.

Self-withdrawal and comorbidities were common reasons for exclusion in our study whereas inadequate ECT was the primary cause of exclusion in the previous study. ${ }^{13}$ This difference between the two studies may relate to the previous study design requiring failure to respond to an adequate course of ECT as a mandatory inclusion criterion. Patients with TRD who responded to a course of ECT, but relapsed due to discontinuation of maintenance ECT ${ }^{9}$ or with partial response to maintenance $\mathrm{ECT}^{32}$ were included. Moreover, several published DBS trials for TRD also included patients who responded to ECT. ${ }^{9,15,16,32}$ In our previous and recent DBS trials, we included patients who responded to ECT but declined to consider maintenance ECT due to memory disturbances or patients who could not tolerate ECT due to post-ECT headaches/confusion. The reasons for inclusion of ECT responders in our DBS trial were based on the evidence that ECT responders were more likely to show response to $\mathrm{DBS}^{32}$ and substantial proportion of ECT responders may relapse requiring maintenance $\mathrm{ECT}^{33}$. Patients may favor DBS over ECT maintenance because of less cognitive side effects with DBS and less opportunity cost in continual need for treatment. Additionally, three patients who never had ECT previously and declined to undertake ECT at the screening stage were included for DBS surgery. In line with participatory model of medical practice, we included those patients who refused to go for ECT based on their personal choice. However, this group accounts for only $13 \%$ of patients who underwent DBS surgery. Our view is that ECT should be recommended prior to DBS and education about the benefits of ECT should be provided to all patients who have never had it. Mandatory ECT exclusion criteria would eliminate several TRD patients who could benefit from DBS for various reasons. At the same time, novel non-invasive treatments for TRD, such as TMS and ketamine infusion, should be considered prior to the selection of patients for future DBS trials in TRD given the invasive and experimental nature of DBS for TRD, and the increasing availability and accessibility of these noninvasive treatments. 
This study has several limitations. As self-referral participants were predominantly recruited through advertisements and television/radio segments this may have caused a volunteer bias which may not reflect TRD patients in the general population. Participants from professional referrals likely were from the academic settings which were given information about the trial and may also not be reflective of TRD patients in general population. This study was conducted in a single academic center reducing generalizability. The results also reflect the screening procedures and eligibility criteria of this study which may also limit the generalizability. For instance, ECT exclusion was not mandatory in our protocol and we used self-report of medical or psychiatric co-morbidity in the initial stages of evaluation which has questionable validity without verifiable objective information from medical or psychiatric records. Almost all of our participants were Caucasians representing the major ethnic demographic of the province of Alberta and therefore our findings cannot be generalized to other ethnic communities. Culturally sensitive recruitment strategies need to be deployed to recruit ethnic minorities.

\section{Conclusions}

While DBS holds promise for treating TRD, there is ongoing need for further DBS trials using different trial designs to demonstrate clinical efficacy and justify the costeffectiveness of this expensive treatment. Our insights into recruitment will inform future DBS trials. Since patient recruitment is a key determinant of success for DBS trials, comprehensive recruitment strategies including professional and self-referral systems should be implemented. Emphasis should be placed on continual engagement of stakeholders (patients and professionals) and effective communication of trial details to improve recruitment. Since DBS will remain experimental for several more years, it should be delivered through research programs affiliated with specialized TRD clinics or neuromodulation units. Integrated research and clinical programs for comprehensive short- and long-term care will encourage mental health professionals to consider DBS as an option for TRD. Our study revealed the importance of self-referral for trial success. This can be further improved by proper preplanning of budget and human resources to reach out to the community through social media and also for screening, reviewing previous psychiatric documents and conducting psychiatric assessments of large numbers of self-referrals. Future DBS trials for TRD should aim to improve recruitment through combined professional and self-referral pathways.

\section{Acknowledgments}

Funding for the study was provided by a grant from Alberta Innovates Health Solutions (AIHS), Alberta, Canada. The funding agency had no role in the study design, collection, analysis and interpretation of data or the preparation, review or approval of the manuscript. Partial data were presented as a poster at the international conference on Brian Stimulation at Barcelona, Spain in March 2017 and as an oral presentation at Alberta Psychiatric Association annual meeting at Banff in March 2018. Maida Khan RN provided assistance in the preparation of the manuscript.

\section{Author Contributions}

All authors made a significant contribution to the conception, study design, execution, and acquisition of data, analysis and interpretation. They participated in drafting, revising or critically reviewing the article. They gave final approval of the version to be published and agreed upon the journal to which the article has been submitted and agreed to be accountable for all aspects of the work. GM was deceased before the completion of the manuscript.

\section{Disclosure}

Dr. Ramasubbu has received honorarium for serving in the advisory committee of Astra Zeneca, Lund beck, Janssen, and Otsuka. He also received investigator-initiated grant from Astra Zeneca and Pfizer. Dr Zelma Kiss reports grants from Alberta Innovates Health Solutions, during the conduct of the study. Other authors declare no potential conflict of interest.

\section{References}

1. Ramasubbu R, Lang S, Kiss ZHT. Dosing of electrical parameters in Deep Brain Stimulation (DBS) for intractable depression: a review of clinical studies. Front Psychiatry. 2018;9:302. doi:10.3389/fpsyt.20 18.00302

2. Holtzheimer PE, Husain MM, Lisanby SH, et al. Subcallosal cingulate deep brain stimulation for treatment-resistant depression: a multisite, randomised, sham-controlled trial. Lancet Psychiatry. 2017;4 (11):839-849. doi:10.1016/S2215-0366(17)30371-1

3. Dougherty DD, Rezai AR, Carpenter LL, et al. A randomized sham-controlled trial of deep brain stimulation of the ventral capsule/ ventral striatum for chronic treatment-resistant depression. Biol Psychiatry. 2015;78(4):240-248. doi:10.1016/j.biopsych.2014.11.023

4. Bari AA, Mikell CB, Abosch A, et al. Charting the road forward in psychiatric neurosurgery: proceedings of the 2016 American society for stereotactic and functional neurosurgery workshop on neuromodulation for psychiatric disorders. J Neurol Neurosurg Psychiatry. 2018;89(8):886-896. doi:10.1136/jnnp-2017-317082

5. Riva-Posse P, Choi KS, Holtzheimer PE, et al. A connectomic approach for subcallosal cingulate deep brain stimulation surgery: prospective targeting in treatment-resistant depression. Mol Psychiatry. 2018;23(4):843-849. doi:10.1038/mp.2017.59 
6. Tsolaki E, Espinoza R, Pouratian N. Using probabilistic tractography to target the subcallosal cingulate cortex in patients with treatment resistant depression. Psychiatry Res. 2017;261:72-74. doi:10.1016/j. pscychresns.2017.01.006

7. Brown EC, Clark DL, Forkert ND, Molnar CP, Kiss ZHT, Ramasubbu R. Metabolic activity in subcallosal cingulate predicts response to deep brain stimulation for depression. Neuropsychopharmacology. 2020;45 (10):1681-1688. doi:10.1038/s41386-020-0745-5

8. Clark DL, MacMaster FP, Brown EC, Kiss ZHT, Ramasubbu R. Rostral anterior cingulate glutamate predicts response to subcallosal deep brain stimulation for resistant depression. J Affect Disord. 2020;266:90-94. doi:10.1016/j.jad.2020.01.058

9. Bergfeld IO, Mantione M, Hoogendoorn ML, et al. Deep brain stimulation of the ventral anterior limb of the internal capsule for treatment-resistant depression: a randomized clinical trial. JAMA Psychiatry. 2016;73 (5):456-464. doi:10.1001/jamapsychiatry.2016.0152

10. Puigdemont D, Portella M, Perez-Egea R, et al. A randomized double-blind crossover trial of deep brain stimulation of the subcallosal cingulate gyrus in patients with treatment-resistant depression: a pilot study of relapse prevention. J Psychiatry Neurosci. 2015;40 (4):224-231. doi:10.1503/jpn.130295

11. Widge AS, Malone DA Jr, Dougherty DD. Closing the loop on deep brain stimulation for treatment-resistant depression. Front Neurosci. 2018;12:175. doi:10.3389/fnins.2018.00175

12. Eitan R, Fontaine D, Benoit M, et al. One year double blind study of high vs low frequency subcallosal cingulate stimulation for depression. J Psychiatr Res. 2018;96:124-134. doi:10.1016/j.jpsychires.2017.09. 026

13. Filkowski MM, Mayberg HS, Holtzheimer PE. Considering eligibility for studies of deep brain stimulation for treatment-resistant depression: insights from a clinical trial in unipolar and bipolar depression. J ECT. 2016;32(2):122-126. doi:10.1097/YCT.000000 0000000281

14. Malone DA Jr, Dougherty DD, Rezai AR, et al. Deep brain stimulation of the ventral capsule/ventral striatum for treatment-resistant depression. Biol Psychiatry. 2009;65(4):267-275. doi:10.1016/j. biopsych.2008.08.029

15. Ramasubbu R, Clark DL, Golding S, et al. Long versus short pulse width subcallosal cingulate stimulation for treatment-resistant depression: a randomised, double-blind, crossover trial. Lancet Psychiatry. 2020;7(1):29-40. doi:10.1016/S2215-0366(19)30415-8

16. Ramasubbu R, Anderson S, Haffenden A, Chavda S, Kiss ZH. Double-blind optimization of subcallosal cingulate deep brain stimulation for treatment-resistant depression: a pilot study. J Psychiatry Neurosci. 2013;38(5):325-332. doi:10.1503/jpn.120160

17. van Beljouw IM, Verhaak PF, Cuijpers P, van Marwijk HW, Penninx BW. The course of untreated anxiety and depression, and determinants of poor one-year outcome: a one-year cohort study. BMC Psychiatry. 2010;10(1):86. doi:10.1186/1471-244X-10-86

18. Boerema AM, Kleiboer A, Beekman AT, van Zoonen K, Dijkshoorn H, Cuijpers P. Determinants of help-seeking behavior in depression: a cross-sectional study. BMC Psychiatry. 2016;16(1):78. doi:10.1186/s12888-016-0790-0

19. Mojtabai R, Olfson M, Mechanic D. Perceived need and help-seeking in adults with mood, anxiety, or substance use disorders. Arch Gen Psychiatry. 2002;59(1):77-84. doi:10.1001/archpsyc.59.1.77
20. Sherwood CSP, Rimes KA, Rimes KA. Help-seeking for depression: the role of beliefs, attitudes and mood. Behav Cogn Psychother. 2007;35(05):541-554. doi:10.1017/S1352465807003815

21. Brown JSL, Murphy C, Kelly J, Goldsmith K. How can we successfully recruit depressed people? Lessons learned in recruiting depressed participants to a multi-site trial of a brief depression intervention (the 'CLASSIC' trial). Trials. 2019;20(1):131. doi:10.1186/ s13063-018-3033-5

22. Dunn LB, Holtzheimer PE, Hoop JG, Mayberg HS, Appelbaum PS. Ethical issues in deep brain stimulation research for treatment-resistant depression: focus on risk and consent. $A J O B$ Neurosci. 2011;2(1):29-36. doi:10.1080/21507740.2010.533638

23. Freedman B. Equipoise and the ethics of clinical research. $N$ Engl J Med. 1987;317(3):141-145. doi:10.1056/NEJM198707163170304

24. Treweek S, Lockhart P, Pitkethly M, et al. Methods to improve recruitment to randomised controlled trials: cochrane systematic review and meta-analysis. BMJ Open. 2013;3(2):e002360. doi:10.1136/bmjopen-2012-002360

25. Ross S, Grant A, Counsell C, Gillespie W, Russell I, Prescott R. Barriers to participation in randomised controlled trials: a systematic review. J Clin Epidemiol. 1999;52(12):1143-1156. doi:10.1016/ S0895-4356(99)00141-9

26. Cormier J, Iorio-Morin C, Mathieu D, Ducharme S. Psychiatric neurosurgery: a survey on the perceptions of psychiatrists and residents. Can J Neurol Sci. 2019;46(3):303-310. doi:10.1017/ cjn. 2019.5

27. Huang GD, Bull J, Johnston McKee K, et al. Clinical trials recruitment planning: a proposed framework from the clinical trials transformation initiative. Contemp Clin Trials. 2018;66:74-79. doi:10.10 16/j.cct.2018.01.003

28. Katz M, Kilbane C, Rosengard J, Alterman RL, Tagliati M. Referring patients for deep brain stimulation: an improving practice. Arch Neurol. 2011;68(8):1027-1032. doi:10.1001/archneurol.2011.151

29. Davidson BZL, Meng Y, Hanani C, Lipsman N. Psychiatric neuromodulation: the underappreciated importance of pre- and post-treatment care. Mol Psychiatry. 2021;26(2):366-369. doi:10.10 38/s41380-020-0851-0

30. Young AH, Juruena MF, De Zwaef R, Demyttenaere K. Vagus nerve stimulation as adjunctive therapy in patients with difficult-to-treat depression (RESTORE-LIFE): study protocol design and rationale of a real-world post-market study. BMC Psychiatry. 2020;20(1):471. doi:10.1186/s12888-020-02869-6

31. Merkl A, Aust S, Schneider GH, et al. Deep brain stimulation of the subcallosal cingulate gyrus in patients with treatment-resistant depression: a double-blinded randomized controlled study and long-term follow-up in eight patients. $J$ Affect Disord. 2018;227:521-529. doi:10.1016/j.jad.2017.11.024

32. Puigdemont D, Perez-Egea R, Portella MJ, et al. Deep brain stimulation of the subcallosal cingulate gyrus: further evidence in treatment-resistant major depression. Int J Neuropsychopharmacol. 2012;15(1):121-133. doi:10.1017/S1461145711001088

33. Kellner CH, Knapp RG, Petrides G, et al. Continuation electroconvulsive therapy vs pharmacotherapy for relapse prevention in major depression: a multisite study from the Consortium for Research in Electroconvulsive Therapy (CORE). Arch Gen Psychiatry. 2006;63 (12):1337-1344. doi:10.1001/archpsyc.63.12.1337 


\section{Publish your work in this journal}

Neuropsychiatric Disease and Treatment is an international, peerreviewed journal of clinical therapeutics and pharmacology focusing on concise rapid reporting of clinical or pre-clinical studies on range of neuropsychiatric and neurological disorders. This journal is indexed on PubMed Central, the 'PsycINFO' database and CAS, and is the official journal of The International Neuropsychiatric Association (INA). The manuscript management system is completely online and includes a very quick and fair peer-review system, which is all easy to use. Visit http://www.dovepress.com/testimonials.php to read real quotes from published authors.

Submit your manuscript here: https://www.dovepress.com/neuropsychiatric-disease-and-treatment-journal 